\title{
Group refractive index calculation by difference approximation for length measurement
}

D. Wei

weidong@mech.nagaokaut.ac.jp

\section{Xiao}

P. Yang

\author{
Department of Mechanical Engineering, Nagaoka University of Technology, Nagaoka City, Niigata \\ 940-2188, Japan \\ School of Mechanical Engineering, Beijing Institute of Technology, Beijing 100081, People's Republic of \\ China \\ Department of Mechanical and Electrical Engineering, School of Aerospace Engineering, Xiamen Uni- \\ versity, Xiamen City, Fujian Province,361006, People's Republic of China
}

In this study, the possibility of employing a difference approximation to facilitate calculation of the group refractive index of air (GRA) was investigated. The forward, backward, and central difference methods were used to numerically approximate the first-order derivatives of the phase refractive index based on the Edlén empirical equations. To confirm the validity of the calculations, the calculated results were compared with the theoretical analysis results and the values in a related paper. It was found that the GRA computation could be easily approximated by the two-point central difference method with a step size of $10 \mathrm{~nm}$.

[DOI: http://dx.doi.org/10.2971/jeos.2016.16013]

Keywords: Group refractive index, difference approximation, frequency comb, length measurement, metrology

\section{INTRODUCTION}

In recent years, femtosecond optical frequency comb (FOFC)based length measurements have gained considerable attention. Among these length measurement methods, adjacent pulse repetition interval length (APRIL)-based measurements [1]-[13] have been demonstrated to be technically feasible. The APRIL is a coherent representation of individual wavelengths. In these experiments, the APRIL was used as the ruler length instead of the wavelengths. In general, lengths measured in air vary depending on the refractive index of the air. In wavelength-based length measurements, the phase refractive index of air (PRA) is used to obtain the length in a vacuum. However, in APRIL-based length measurements, the group refractive index of air (GRA) must be calculated to determine the length in a vacuum.

As discussed in the Methods section, the GRA can be calculated by taking the derivative of the PRA. In practice, it is technically difficult to obtain GRA values due to the complexity of the differentiation of the empirical formula. Not all non-expert users are skilled at performing differential calculations.

It is well known that differentiation can be approximated by difference approximation [14, 15]. Reliable PRA calculators (for example, [16]) that can provide PRA values for specific wavelengths under specified environmental conditions exist on the internet.

Based on these two facts, in this paper, we discuss the possibility of using difference approximation methods to calculate GRAs. Difference approximations were performed on an empirical equation, and the calculated results were compared with the theoretical results as well as with the values given in a related paper. Based on our results, we concluded that the two-point central difference method is as an acceptable means of numerically approximating GRAs. To the best of the authors' knowledge, the determination of specific GRA values based on difference approximation methods has not been investigated previously. We expect to be able to contribute to the accessibility of GRA based on four arithmetic operations for non-expert users.

This paper is organized as follows. First, we recall the basic difference approximation principles in Section 2. The numerical calculations are described in Section 3. Finally, the main conclusions are given in Section 4.

\section{METHODS}

In general, a GRA can be calculated as follows [17]:

$$
\begin{aligned}
\mathbf{n}_{\mathrm{g}}\left(\lambda_{\text {cen_vac }}\right)= & \mathbf{n}_{\mathrm{p}}\left(\lambda_{\text {cen_vac }}\right)-\lambda_{\text {cen_vac }} \\
& \times\left(\mathbf{d} \mathbf{n}_{\mathrm{p}}\left(\lambda_{\text {vac }}\right) / \mathbf{d} \lambda_{\text {vac }}\right) \lambda_{\text {cen_vac }}
\end{aligned}
$$

where $\lambda_{\text {vacis }}$ the wavelength in a vacuum, $n_{p}\left(\lambda_{\text {Vac }}\right)$ is the PRA, $\lambda_{\text {cen_vac }}$ is the central wavelength of the FOFC, and $\left(\mathrm{d} n_{p}\left(\lambda_{\mathrm{Vac}}\right) / d \lambda_{\mathrm{Vac}}\right)_{\lambda_{\text {cen }} \mathrm{vac}}$ is the derivative of the function $y=n_{p}\left(\lambda_{\mathrm{Vac}}\right)$ at $\lambda_{\mathrm{vac}}=\lambda_{\text {cen_vac. }}$ More details about the FOFC can be found in Ref. [18]. To calculate PRAs, different empirical formulas [16], [19]-[21] are often used. Because of the limit on the length of this paper, we only discuss the Edlen empirical equations herein. In the Edlén empirical equations $[16,20,21]$, which were the focus of this study, a PRA can be 
derived from the wavelength $\lambda_{\mathrm{vac}}$, temperature $T$, barometric pressure $P$, and humidity $H$ as $n_{p}=f\left(\lambda_{\mathrm{vac}}, T, P, H\right)$.

In general, derivatives can be approximated by using difference approximations $[14,15]$. Three methods of numerically approximating first-order derivatives are often used, namely, the two-point forward $n_{\text {p_forw }}^{\prime}\left(\lambda_{\mathrm{Vac}}\right)$, two-point backward $n_{\text {p_backw }}^{\prime}\left(\lambda_{\mathrm{Vac}}\right)$, and two-point central difference $n_{\mathrm{p} \_c e n t}^{\prime}\left(\lambda_{\mathrm{vac}}\right)$ methods. The difference approximation equations can be found in Refs. [14] and [15].

One of the goals of this paper is to qualify the value of the wavelength interval $\Delta \lambda$ of the difference approximations required to ensure the accuracy of the approximation.

\section{NUMERICAL CALCULATIONS AND RESULTS}

A series of numerical calculation methods were investigated to determine which could be used to simplify the GRA calculation procedure. Specifically, the forward, backward, and central difference methods were used to numerically approximate the first-order derivatives of the PRA to identify which yielded the best accuracy. To confirm the validity of the calculations, we compared the calculated results with the results of theoretical analysis and the values given in a related paper.

The first numerical calculations were performed using $\lambda_{\text {vac }}=1560 \mathrm{~nm}$ with the following environmental conditions: $T=20^{\circ} \mathrm{C}, P=101.325 \mathrm{kPa}$, and $H=50 \%$. In Fig. 1, the absolute value of difference, which is the absolute value of the difference between the exact (theoretical) value $\left(n^{\prime}(1560)\right)$ and the approximate value, is plotted against the step size $\Delta \lambda$. To obtain this plot, we used the phase refractive index equations given in Ref. [16]. The formula used to perform the calculations can be easily accessed via the internet [16]. Based on their definitions, the approximate values of $n_{\text {p_forw }}^{\prime}(1560), n_{\text {p_backw }}^{\prime}(1560)$, and $n_{\text {p_cent }}^{\prime}(1560)$ were calculated for different $\Delta \lambda$ values. The exact value of $n^{\prime}$ (1560) was calculated by obtaining $n^{\prime}\left(\lambda_{\mathrm{Vac}}\right)$ using differential calculus [22] and substituting $\lambda_{\mathrm{Vac}}=1560 \mathrm{~nm}$ and was determined to be 1.000269395 . The analytical differential calculus formula is complex. To access the program code for free, please contact the corresponding author via e-mail.

It is well known that forward and backward difference approximations are accurate to order $\Delta \lambda$. The central difference approximation is thus accurate to order $(\Delta \lambda)^{2}$. Therefore, for small values of $\Delta \lambda$, central approximations will generally be more accurate than either forward or backward approximations. Our calculated results are consistent with this tendency. As shown in Figure 1 , as $\Delta \lambda$ decreases, the absolute value of the difference increases. This trend is apparent because the PRA values were calculated as decimals and rounded to nine digits. For example, via Ref. [16], we obtained $n_{p}(1560)=1.000268147, n_{p}(1561)=1.000268146$, $n_{p}(1559)=1.000268148, n_{p}(1560.1)=1.000268147$, $n_{p}(1559.9)=1.000268147, n_{p}(1560.01)=1.000268147$, and $n_{p}(1559.99)=1.000268147$. The accuracies of these

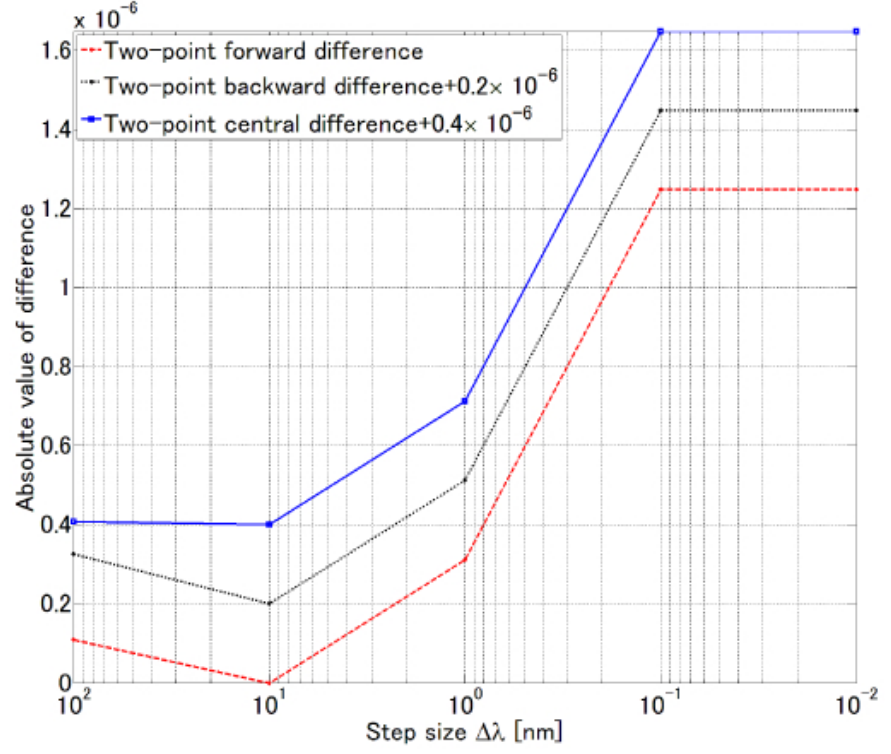

FIC. 1 Change in absolute value of difference with step size $\Delta \lambda$. Vertical origins of plots of two-point backward difference and two-point central difference are shifted for clarity.

values were limited by the rounding error; thus, the precision for intervals smaller than $10 \mathrm{~nm}$ decreased. When $\Delta \lambda=0.1$ or $0.01 \mathrm{~nm}$, there is no difference between $n_{p}(\lambda+\Delta \lambda)$ and $n_{p}(\lambda-\Delta \lambda)$. The approximate values of $n_{\mathrm{p} \text { forw }}^{\prime}(1560)$, $n_{\text {p_backw }}^{\prime}(1560)$, and $n_{\text {p_cent }}^{\prime}(1560)$ were calculated to be the same.

From Figure 1, it can be concluded that the absolute value of difference is the smallest for $\Delta \lambda=10$. The slope of the GRA varies depending on $\lambda_{\mathrm{Vac}}, T, P$, and $H$. Therefore, we compared the theoretical analysis results and two-point differences for other frequencies under different environmental conditions with $\Delta \lambda=10 \mathrm{~nm}$.

Figure 2 shows the variation of the absolute value of the difference with wavelength $\left(\lambda_{\mathrm{Vac}} \in[310,1690] \mathrm{nm}\right)$ with $\Delta \lambda=10 \mathrm{~nm}, T=20^{\circ} \mathrm{C}, P=101.325 \mathrm{kPa}$, and $H=50 \%$. Since the absolute value of the difference is the largest at $310 \mathrm{~nm}$, we address only the case of $\lambda_{\mathrm{Vac}}=310 \mathrm{~nm}$ in the following discussion.

The changes in absolute value of the difference with temperature $\left(T \in[10,30]{ }^{\circ} \mathrm{C}\right)$ are shown in Figure 3. With increasing temperature, the absolute value of difference decreases. Therefore, in the following, we present only the results for $\mathrm{T}=10^{\circ} \mathrm{C}$.

The changes in the absolute value of the difference with pressure $(P \in[60,120] \mathrm{kPa})$ are shown in Figure 4 . With increasing pressure, the absolute value of the difference increases. Therefore, in the following, we address only the case of $P=120 \mathrm{kPa}$.

The variation of the absolute value of the difference with humidity $(H \in[5,85] \%)$ is shown in Figure 5. As can been seen in Figures 2-5, the maximum absolute value of the difference is about $1.5 \times 10^{-7}$. We also performed calculations in two specific ranges: the visible light range, $380-770 \mathrm{~nm}$, and the opti- 
(a)

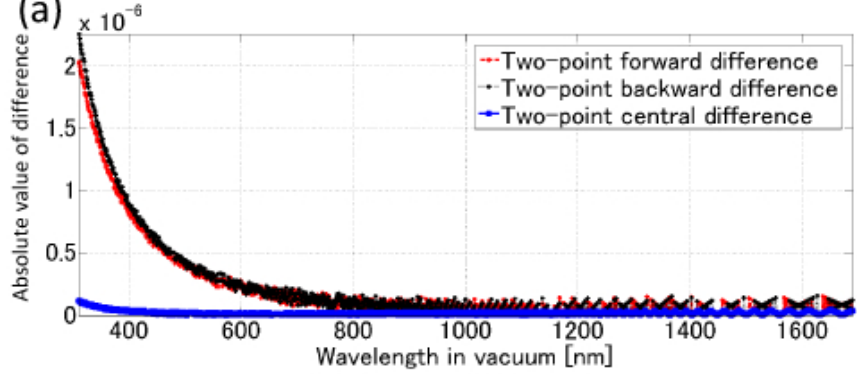

(b)

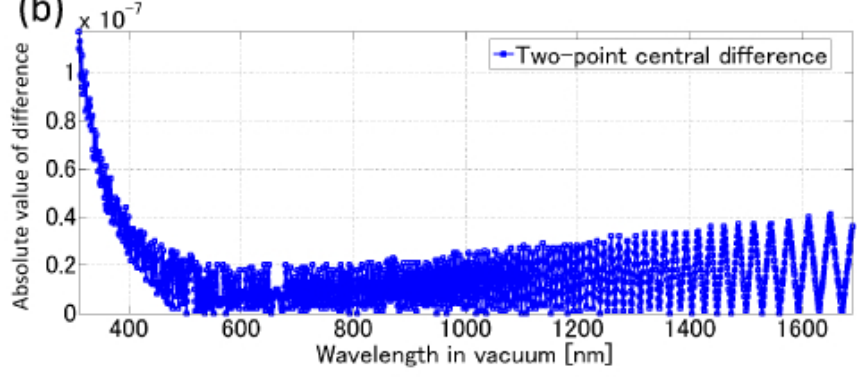

FIG. 2 (a) Variation of absolute value of difference with wavelength for $\Delta \lambda=10 \mathrm{~nm}$ ( $T=20{ }^{\circ} \mathrm{C}, P=101.325 \mathrm{kPa}, H=50 \%$ ). (b) Zoomed-in view of variation of absolute value of difference based on two-point central difference method.
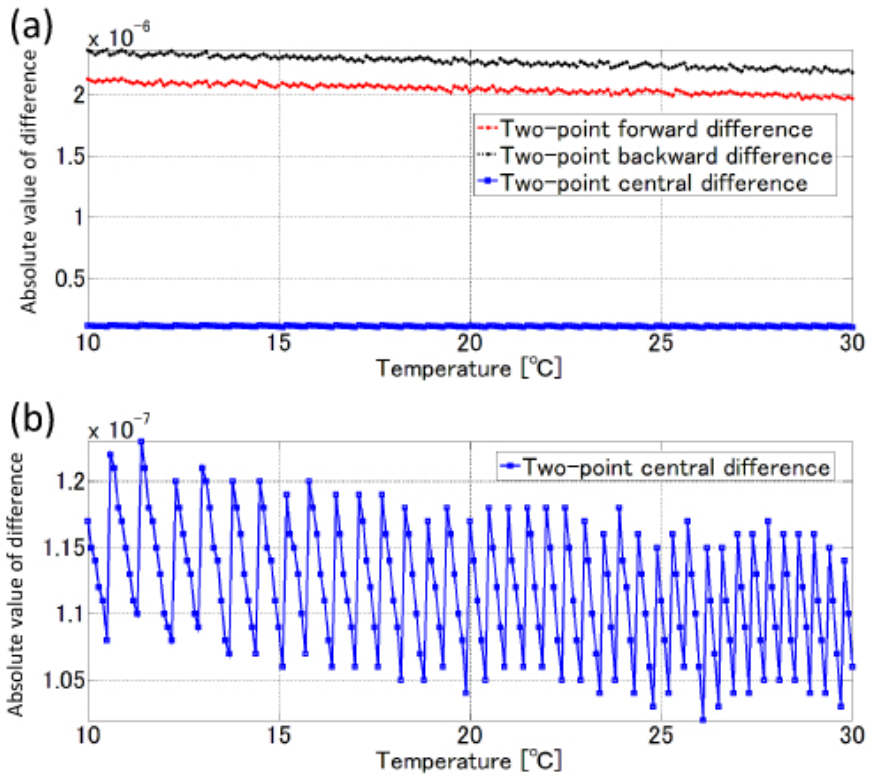

FIG. 3 (a) Variation of absolute value of difference with temperature for $\Delta \lambda=10 \mathrm{~nm}$ $\left(\lambda_{\text {vac }}=310 \mathrm{~nm}, P=101.325 \mathrm{kPa}, H=50 \%\right.$ ). (b) Zoomed-in view of variation of absolute value of difference based on two-point central difference method.

cal fiber transmittance range, $1260-1625 \mathrm{~nm}$. In the visible and optical fiber transmittance ranges, the maximum absolute values of the differences are less than $60 \times 10^{-9}$ and $40 \times 10^{-9}$, respectively. It is well known that the Edlén empirical equations have an uncertainty contribution of $30-50 \times 10^{-9}$ [16]. By comparing the two maximum absolute values of the differences with this value, we consider that they are acceptable.

In each of Figures 2-5, the variation of the absolute value of the difference forms a zigzag. This pattern originates from the PGA calculations [16], in which the decimal results were rounded to nine digits.
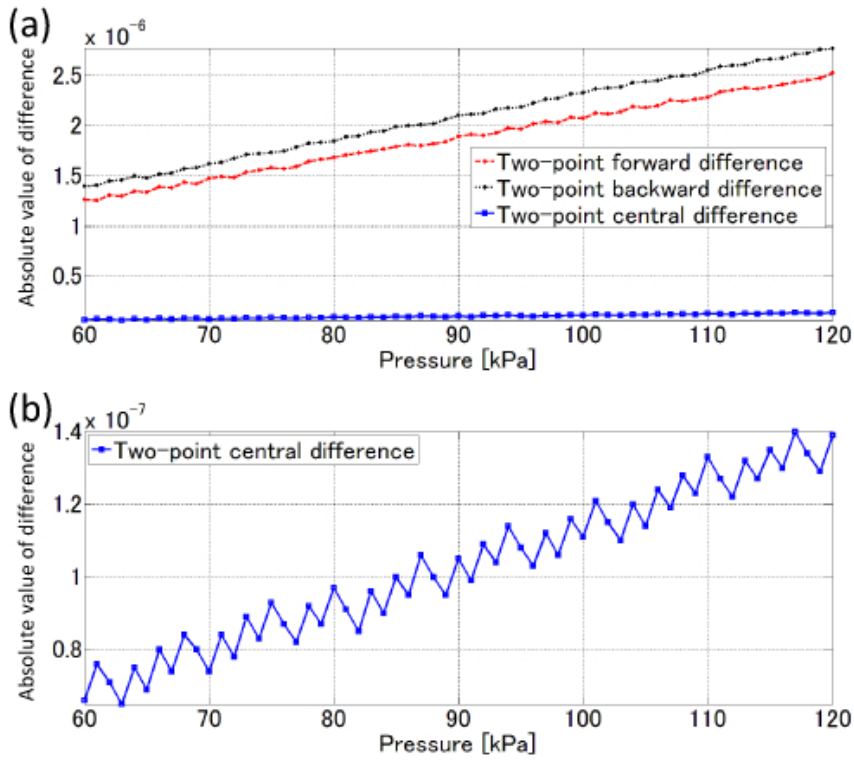

FIG. 4 (a) Variation of absolute value of difference with pressure for $\Delta \lambda=10 \mathrm{~nm}$ $\left(\lambda_{\text {vac }}=310 \mathrm{~nm}, \mathrm{~T}=10^{\circ} \mathrm{C}, H=50 \%\right.$ ). (b) Zoomed-in view of variation of absolute value of difference based on two-point central difference method.
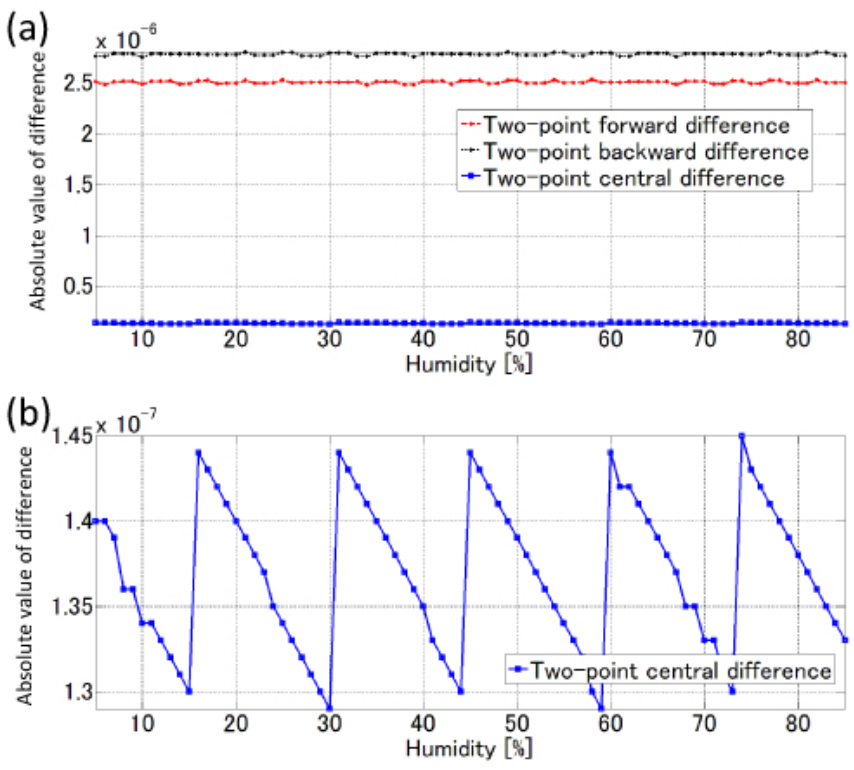

FIG. 5 (a) Variation of absolute value of difference with humidity for $\Delta \lambda=10 \mathrm{~nm}$ $\left(\lambda_{\text {vac }}=310 \mathrm{~nm}, T=10^{\circ} \mathrm{C}, P=120 \mathrm{kPa}\right)$. (b) Zoomed-in view of variation of absolute value of difference based on two-point central difference method.

By using Richardson extrapolation, the approximation error could be further reduced. Since the two-point central difference method provides the expected approximation accuracy, we did not estimate the value of the derivative by using more than two data points. In conclusion, the results indicate that the two-point central difference method can be used to obtain acceptable approximations for GRAs with $\Delta \lambda=10 \mathrm{~nm}$.

By comparing the two-point central difference results with those of Ref. [22], we further confirmed the validity of the calculations. Based on Ref. [22], the GRAs for wavelengths between $2000 \AA$ and $21,000 \AA$ at $15{ }^{\circ} \mathrm{C}, 760 \mathrm{~mm} \mathrm{Hg}$, and $0.03 \%$ 


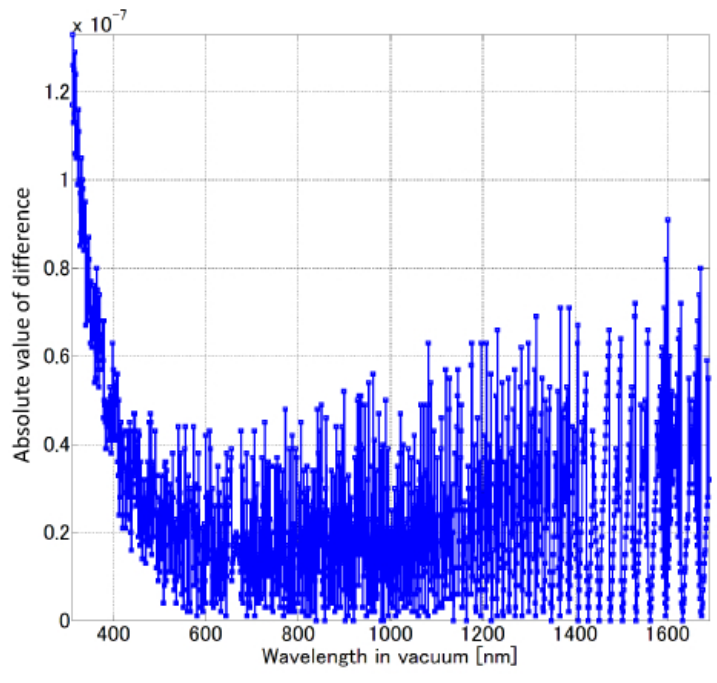

FIG. 6 Comparison of results calculated using two-point central difference method and Eq. (2).

$\mathrm{CO}_{2}$ can be determined by using Eq. (2):

$$
\begin{aligned}
(n \mathrm{~g}-1) \times 10^{6}= & 83.4213+\frac{24060.30}{\left(130-\sigma^{2}\right)^{2}}\left(130+\sigma^{2}\right) \\
& +\frac{159.97}{\left(38.9-\sigma^{2}\right)^{2}}\left(38.9+\sigma^{2}\right)
\end{aligned}
$$

where $\sigma=10^{6} / \lambda$. The GRA was calculated both by Eq. (2) and by using the two-point central difference method with a step size of $\Delta \lambda=10 \mathrm{~nm}$ for comparison. Figure 6 shows the difference between the results obtained using these two methods.

\section{CONCLUSION}

We performed numerical experiments to examine the feasibility of calculating GRAs by using the two-point forward, two-point backward, and two-point central difference approximation methods. Under environmental conditions of $T \in[10,30]{ }^{\circ} \mathrm{C}, P \in[60,120] \mathrm{kPa}$, and $H \in[5,85] \%$, we confirmed the validity of the difference approximations. We determined that GRA computation can be easily approximated by the two-point central difference method and can achieve approximation accuracies of better than $60 \times 10^{-9}$ in both the visible light range $(380-770 \mathrm{~nm})$ and the optical fiber light transmittance range $(1260-1625 \mathrm{~nm})$ with $\Delta \lambda=10 \mathrm{~nm}$. The understanding obtained in this study will facilitate GRA calculation for non-expert users by reducing the required calculations to involve just the four arithmetic operations.

\section{ACKN OWLEDGEMENTS}

This research work was partially financially supported by a research grant from the Intelligent Cosmos Academic Foundation (2015-4).

\section{References}

[1] L. Xu, C. Spielmann, A. Poppe, T. Brabec, F. Krausz, and T. W. Hansch, "Route to phase control of ultrashort light pulses," Opt. Lett. 21, 2008-2010 (1996).
[2] A. M. Chekhovsky, A. N. Golubev, and M. V. Gorbunkov, "Optical pulse distance-multiplying interferometry," Appl. Optics 37, 34803483 (1998).

[3] Y. Yamaoka, K. Minoshima, and H. Matsumoto, "Direct Measurement of the Group Refractive Index of Air with Interferometry between Adjacent Femtosecond Pulses," Appl. Optics 41, 4318-4324 (2002).

[4] J. Ye, "Absolute measurement of a long, arbitrary distance to less than an optical fringe," Opt. Lett. 29, 1153-1155 (2004).

[5] M. Cui, R. N. Schouten, N. Bhattacharya, and S. A. Berg, "Experimental demonstration of distance measurement with a femtosecond frequency comb laser," J. Eur. Opt. Soc.-Rapid 3, 08003 (2008).

[6] H. Matsumoto, X. Wang, K. Takamasu, and T. Aoto, "Absolute Measurement of Baselines up to $403 \mathrm{~m}$ Using Heterodyne Temporal Coherence Interferometer with Optical Frequency Comb," Appl. Phys. Express 5, 046601 (2012).

[7] C. Narin, T. Satoru, T. Kiyoshi, and M. Hirokazu, “A new method for high-accuracy gauge block measurement using $2 \mathrm{CHz}$ repetition mode of a mode-locked fiber laser," Meas. Sci. Technol. 23, 054003 (2012).

[8] X. Wang, S. Takahashi, K. Takamasu, and H. Matsumoto, "Space position measurement using long-path heterodyne interferometer with optical frequency comb," Opt. Express 20, 2725-2732 (2012).

[9] X. Wang, S. Takahashi, K. Takamasu, and H. Matsumoto, "Spatial positioning measurements up to $150 \mathrm{~m}$ using temporal coherence of optical frequency comb," Precis. Eng. 37, 635-639 (2013).

[10] D. Wei, K. Takamasu, and H. Matsumoto, "A study of the possibility of using an adjacent pulse repetition interval length as a scale using a Helium-Neon interferometer," Precis. Eng. 37, 694698 (2013).

[11] D. Wei, and M. Aketagawa, "Comparison of length measurements provided by a femtosecond optical frequency comb," Opt. Express 22, 7040-7045 (2014).

[12] W. Sudatham, H. Matsumoto, S. Takahashi, and K. Takamasu, "Verification of the positioning accuracy of industrial coordinate measuring machine using optical-comb pulsed interferometer with a rough metal ball target," Precis. Eng. 41, 63-67 (2015).

[13] W. Sudatham, H. Matsumoto, S. Takahashi, and K. Takamasu, "Non-contact measurement technique for dimensional metrology using optical comb," Measurement 78, 381-387 (2016).

[14] R. Butt, Introduction to Numerical Analysis Using MATLAB® (Jones it Bartlett Learning, Burlington, 2009).

[15] R. Canale, and S. Chapra, Numerical Methods for Engineers (McGraw-Hill Education, New York City, 2014).

[16] J. A. Stone, and J. H. Zimmerman, "Refractive index of air calculator," http://emtoolbox.nist.gov/Wavelength/Edlen.asp.

[17] B. E. A. Saleh, and M. C. Teich, Fundamentals of photonics (WileyInterscience, Hoboken, 2007).

[18] J. Ye, and S. T. Cundiff, Femtosecond optical frequency comb : principle, operation, and applications (Springer, New York, 2005).

[19] P. E. Ciddor, "Refractive index of air: new equations for the visible and near infrared," Appl. Optics 35, 1566-1573 (1996).

[20] K. P. Birch, and M. J. Downs, "An Updated Edlén Equation for the Refractive Index of Air," Metrologia 30, 155 (1993).

[21] E. Bengt, "The Refractive Index of Air," Metrologia 2, 71 (1966).

[22] L. E. Wood, and M. C. Thompson, "The Group Refractive Index of Air," Appl. Optics 7, 1408-1409 (1968). 